\title{
Ionic Liquid-Based Aqueous Biphasic Systems-A Facile Approach for Ionic Liquid Regeneration from Crude Plant Extracts
}

\author{
Rozalina Keremedchieva, Ivan Svinyarov and Milen G. Bogdanov * \\ Faculty of Chemistry and Pharmacy, Sofia University "St. Kl. Ohridski”, 1 J. Bourchier blvd., \\ 1164 Sofia, Bulgaria; E-Mails: rose_lin@abv.bg (R.K.); ivansvinyarov@abv.bg (I.S.) \\ * Author to whom correspondence should be addressed; E-Mail: mbogdanov@chem.uni-sofia.bg; \\ Tel.: +359-2-81-61-228; Fax: +359-2-96-25-438.
}

Academic Editor: Michael Henson

Received: 22 August 2015 / Accepted: 21 November 2015 / Published: 26 November 2015

\begin{abstract}
The possibility for simultaneous controlled partition of the biologically active alkaloid $S$-(+)-glaucine, presented in a crude ionic liquid-aqueous plant extract, and separation of the ionic liquid (IL) employed by means of ionic liquid-based aqueous biphasic systems (IL-ABS) was investigated in this study. The ABS were formed by the addition of inorganic salts with distinct water-structuring properties such as $\mathrm{Na}_{2} \mathrm{CO}_{3}, \mathrm{MgSO}_{4}$, $\left(\mathrm{NH}_{4}\right)_{2} \mathrm{SO}_{4}$, and $\mathrm{NaH}_{2} \mathrm{PO}_{4}$ to an enriched of glaucine extract of Glaucium flavum $\mathrm{Cr}$. (Papaveraceae). The influence of the salt type and concentration on the phase-forming ability, as well as the $\mathrm{pH}$ value on the partition of glaucine between the two phases formed, was comprehensively studied. It was found that the target alkaloid is predominantly transferred into the IL-rich phase, regardless the influential factors. The results obtained were further used as a platform for the development of an improved extractive procedure, ensuring simultaneous glaucine recovery, IL recycling and water removal in a single technological step. Thus, based on the formation of a three-phase system consisting of butyl acetate, IL-rich phase, and salt-rich phase, nearly quantitative recovery of glaucine $(>99 \%)$, IL recycling ( $c a .90 \%)$, and water removal (ca. 85\%) were achieved by salt-induced separation of the crude plant extract.
\end{abstract}

Keywords: ionic liquids; IL-ABS; liquid-liquid extraction; natural compounds; alkaloids 


\section{Introduction}

Recent requirements for the development of green and sustainable technologies constantly increase the interest in research aiming at the substitution of volatile, toxic and flammable conventional molecular solvents with more benign ones [1]. Among the others, ionic liquids (ILs) have received considerable attention in the last decade and were introduced as an alternative liquid medium in numerous processes [2,3]. Particularly, ILs have proved to be efficient solvents for the recovery of a vast number of natural products from plant materials [4,5], providing enhanced extraction yields and significant reduction of the solvent consumption and extraction times in all cases. It is noteworthy, however, that the majority of the studies had been focused on the initial solid-liquid extraction step and neither have attempts for IL recycling - an important issue that addresses the economics of their use- have been undertaken, nor have approaches for isolation of the target compounds in pure form at the end of the process been offered. A short retrospection on the recent literature shows that the choice of a method to be employed depends on the specific properties of both ILs and solutes of interest. For instance, an anti-solvent-induced precipitation proved successful for the isolation of neutral compounds [6-8] and hydrodistillation for the recovery of volatile compounds [9]. Additionally, back-extraction with organic solvents [10-14], partitioning in IL-based aqueous biphasic systems (IL-ABS) [15,16], and in some cases, ion exchange resin [17,18] or resin for selective trapping [19], had given satisfactory results. The lack of systematic studies on the above issues suggests that additional investigations for the establishment of some general rules for the isolation of different classes of natural compounds from IL-based plant extracts have to be performed.

$S$-(+)-Glaucine is the main alkaloid component in the Glaucium flavum Crantz (Papaveraceae). This compound belongs to the group of aporphine alkaloids ( $c f$. Figure 1) and possesses a wide variety of pharmacological effects including antitussive, antinociceptive, anticonvulsant and neuroleptic-like activities [20-23]. Moreover, in combination with some other biologically active components, glaucine enters into the composition of antitussive drugs produced by various manufacturers. In our recent studies $[10,11,24]$, we demonstrated that methanol, widely applied in industry as a solvent to recover value-added chemicals from their natural matrices, can be successfully substituted by 1-butyl-3-methylimidazolium acesulfamate $\left\{\left[\mathrm{C}_{4} \mathrm{C}_{1}\right.\right.$ im $][$ Ace $\left.]\right\}$-aqueous solution in the extraction of the biologically active alkaloid $S$-(+)-glaucine ( $c f$. Figure 1) from aerial parts of $G$. flavum, and the beneficial effect of the IL-based system was attributed not only to the stronger dissolving power of ILs [10] but rather to the pronounced solvent-matrix interactions leading to an IL-induced plant matrix disruption and permeability modification [24]. The protocol developed [11] possesses several advantages in comparison with the conventional one in terms of extraction yield and time, equipment, energy consumption, and number of technological steps, and allows glaucine to be isolated in high purity and satisfactory yield at the end of the process as hydrobromide salt, the latter being its marketed form. Nevertheless, it still requires chloroform (a molecular solvent with proven toxicity to humans) to be employed in the liquid-liquid extraction step prior to glaucine isolation and this can be considered as a shortcoming from a safety standpoint. Furthermore, the protocol proposed for the recovery of IL required the evaporation of water ( $c a .70 \mathrm{wt} . \%$ ) under reduced pressure, the latter accounting for the high price of similar industrial processes [25]. With regard to that, in this study we report data for the partition of glaucine presented in an enriched ionic liquid-aqueous crude extract of G. flavum in IL-ABS. This study aims to check the possibility for the simultaneous separation of the IL from crude plant extract and the 
purification of glaucine by means of its controlled distribution between two aqueous-based phases, thereby avoiding the use of harmful molecular solvents. The ABS were formed by addition of inorganic salts with distinct water-structuring properties such as $\mathrm{Na}_{2} \mathrm{CO}_{3}, \mathrm{MgSO}_{4},\left(\mathrm{NH}_{4}\right)_{2} \mathrm{SO}_{4}$, and $\mathrm{NaH}_{2} \mathrm{PO}_{4}$ to a crude IL-aqueous plant extract, the latter allowing the influence of the salt type and concentration on the phase-forming ability as well as the $\mathrm{pH}$ value on the partition of glaucine between the two phases to be comprehensively studied.
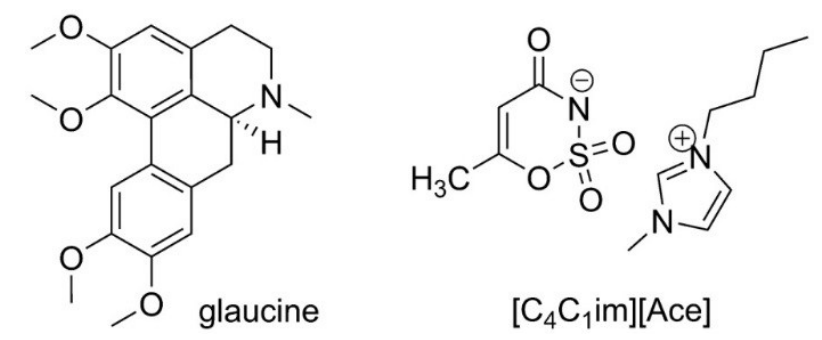

Figure 1. Structures of glaucine and $\left[\mathrm{C}_{4} \mathrm{C}_{1}\right.$ im $][$ Ace $]$.

\section{Experimental Section}

\subsection{Chemicals}

All chemicals used in this study were purchased from Sigma-Aldrich (FOT, Bulgaria). The organic solvents were of analytical grade and acetonitrile used for HPLC analysis was of chromatographic grade. The IL used for extraction experiments was 1-butyl-3-methylimidazolium acesulfamate $\left(\left[\mathrm{C}_{4} \mathrm{C}_{1}\right.\right.$ im] $]$ Ace $]$, Figure 1) and was synthesized, purified and characterized by the authors according to a recently published protocol for the synthesis of hydrophilic ILs [26]. Its structure and purity was unequivocally proven by means of ${ }^{1} \mathrm{H}$ - and ${ }^{13} \mathrm{C}-\mathrm{NMR}$ spectral analysis [10] with a Bruker DRX $250 \mathrm{NMR}$ spectrometer operating at 250.13/62.5 MHz, respectively.

Aerial parts of plant material of Glaucium flavum Cr. (particle size $0.25-0.40 \mathrm{~mm}$ ) and standard sample of glaucine were available from previous studies in our laboratory. Crude plant extract, being a stock solution for the recovery studies (glaucine concentration $=4.0 \mathrm{mg} / \mathrm{mL}$ ), was obtained by means of consecutive extractions of same batch sample [11].

\subsection{Glaucine Quantification}

Glaucine quantification was carried out by means of reverse-phase high performance liquid chromatographic analyses (RP-HPLC) on a GBC liquid chromatography system (GBC Scientific Equipment, Melbourne, Australia), equipped with a LC 1100 HPLC pump, a variable LC 1200 UV/Vis detector, a LC 1431 system organizer, an injector with a $20 \mu \mathrm{L}$ loop and N2000 Chromatostation software (Zhejiang University, PRC, Hangzhou, China)for data treatment. A ZORBAX Extend-C18 (Agilent Technologies, Santa Clara, US; $150 \times 4.6 \mathrm{~mm}$ i.d., $5 \mu \mathrm{m}$ ) was used as an analytical column. The mobile phase was a mixture of $0.1 \%$ triethylamine aqueous solution and acetonitrile (50:50) delivered at a flow rate of $1 \mathrm{~mL} / \mathrm{min}$ [24]. The UV detection wavelength was set up at $280 \mathrm{~nm}$, where aporphine alkaloids have an optimum absorption. Each injection volume was $20 \mu \mathrm{L}$ and the column temperature was ambient. Under these conditions the target alkaloid glaucine was baseline separated and its peak 
was symmetrical. The peak identification was achieved by a comparison of its retention time with the corresponding peak in a standard solution, and glaucine concentration was calculated according to a previously developed relationship. For all analyses, aliquots were taken and were diluted with a certain volume of acetonitrile/water mixture, in order to fit into the linear range of the standard curve, then were filtered through a $0.45 \mu \mathrm{m}$ microporous membrane prior to analysis and were directly injected into the HPLC apparatus. All HPLC analyses were performed in triplicate and the mean value was adopted.

\subsection{Partitioning of Glaucine in IL-Based ABS and Calculations}

Four salting-out agents, namely $\mathrm{Na}_{2} \mathrm{CO}_{3},\left(\mathrm{NH}_{4}\right)_{2} \mathrm{SO}_{4}, \mathrm{MgSO}_{4}$ and $\mathrm{NaH}_{2} \mathrm{PO}_{4}$, were utilized to induce the formation of $\left[\mathrm{C}_{4} \mathrm{C}_{1}\right.$ im] $]$ Ace]-based ABS. The phase diagrams were not established for these systems since the IL concentration is initially restricted to be $1 \mathrm{M}(\mathrm{ca} .30 \%)$. The experiments were performed at ambient conditions in $15 \mathrm{~mL}$ centrifuge tubes, by portion-wise addition of a certain amount of the corresponding salts to $8 \mathrm{~mL}$ of the crude glaucine extract (for details see Table $\mathrm{S} 1$ in Supporting Materials). The mixtures were vigorously shaken to ensure the complete salt dissolution, and next portions were subsequently added until the two-phase region was approached. The samples were then allowed to equilibrate overnight, and in order to form two clearly distinguishable phases, the samples were centrifuged for 5 min prior to analysis. In the majority of the cases, with the exception of $\left(\mathrm{NH}_{4}\right)_{2} \mathrm{SO}_{4}$, the IL-rich phase was formed as the top-phase. The volume of each phase was then detected and aliquots were subjected to analysis as described in Section 2.2.

The extraction efficiency $(E E \%)$ in the liquid-liquid extraction experiments was established by taking into account the corresponding phase ratio ( $R$, Equation (1)) and partition coefficient $(K$, Equation (2)) of glaucine between the two phases. The equations used were as follows: $(i)$ the phase ratio $(R)$ was defined as

$$
R=\frac{V_{\mathrm{IL}}}{V_{\mathrm{Salt}}}
$$

where $V_{\text {IL }}$ and $V_{\text {Salt }}$ refer to the volume of the IL-rich phase and salt-rich phase, respectively; (ii) the partition coefficient $(K)$ was defined as

$$
K=\frac{C_{\mathrm{IL}}}{C_{\mathrm{Salt}}}
$$

where $C_{\mathrm{IL}}$ and $C_{\text {Salt }}$ are the concentration of glaucine in the IL-rich phase and salt-rich phase, respectively; and (iii) the extraction efficiency (EE\%) was determined according Equation (3):

$$
E E \%=\frac{K}{(1+1 / R)} \times 100
$$

\section{Results and Discussion}

It was recently shown by us [11] that glaucine can be recovered from IL-based extracts by means of a classical extraction procedure with chloroform; however, this molecular solvent possesses proven toxicity to humans and its use can be considered as a shortcoming from a safety standpoint. Therefore, we were interested in exploring an alternative possibility for its recovery from the crude plant extract by means of IL-based aqueous two-phase systems (IL-ABS). It was recently shown that aqueous solutions 
of hydrophilic ILs can be switched into IL-ABS by introduction of an inorganic salt with certain water-structuring properties [27]. The driving force of the process can be attributed to the salting-out effect of the inorganic salt, resulting in the formation of two water-rich immiscible phases, where the upper phase (in the majority of the cases) consists mainly of IL and the lower one of concentrated inorganic salt. This way, IL-ABS can be considered as an attractive alternative to the widely applied conventional liquid-liquid extraction with organic solvents, since they ensure simultaneous enrichment, separation, purification and isolation of a particular molecule. Indeed, IL-ABS have been utilized to extract a wide range of compounds such as proteins [28], saccharides [29], enzymes [30], antibiotics [31], phenolic compounds [15], and alkaloids [32,33], to name just a few. It is noteworthy that, in some cases, a controlled partition of the solutes of interest had been achieved by variation of the system composition or its $\mathrm{pH}$ value, thus broadening the scope of IL-ABS applications. Considering these facts, it can be suggested that the recovery of value-added chemicals from raw plant extracts by means of IL-ABS is worthy of investigation.

Four salting-out agents $\left(\mathrm{Na}_{2} \mathrm{CO}_{3}, \mathrm{NaH}_{2} \mathrm{PO}_{4}, \mathrm{MgSO}_{4}\right.$ and $\left.\left(\mathrm{NH}_{4}\right)_{2} \mathrm{SO}_{4}\right)$ were utilized to induce the formation of the two phases in the present study. These salts were chosen in a way to ensure different $\mathrm{pH}$ values in the system, the latter allowing the influence of this factor on the glaucine partition to be assessed. On the other hand, the presence of different inorganic ions with distinct solvation properties in the system suggests that the glaucine partition can be manipulated since different types of interactions can be expected [34]. Furthermore, the salts' impact on the phase ratio and glaucine partition was evaluated by increasing their concentration until saturation is reached. The phase diagrams were not established for the systems under study since the IL concentration is initially restricted to be $1 \mathrm{M} \mathrm{(ca.30 \% )}$ and is in the range of those typically adopted in the majority of the model IL-ABS reported in the literature. The experiments were performed as described in Section 2.3 and the results for the $E E \%$ are summarized in Figure 2 (for details see Table S1 in Supporting Materials).

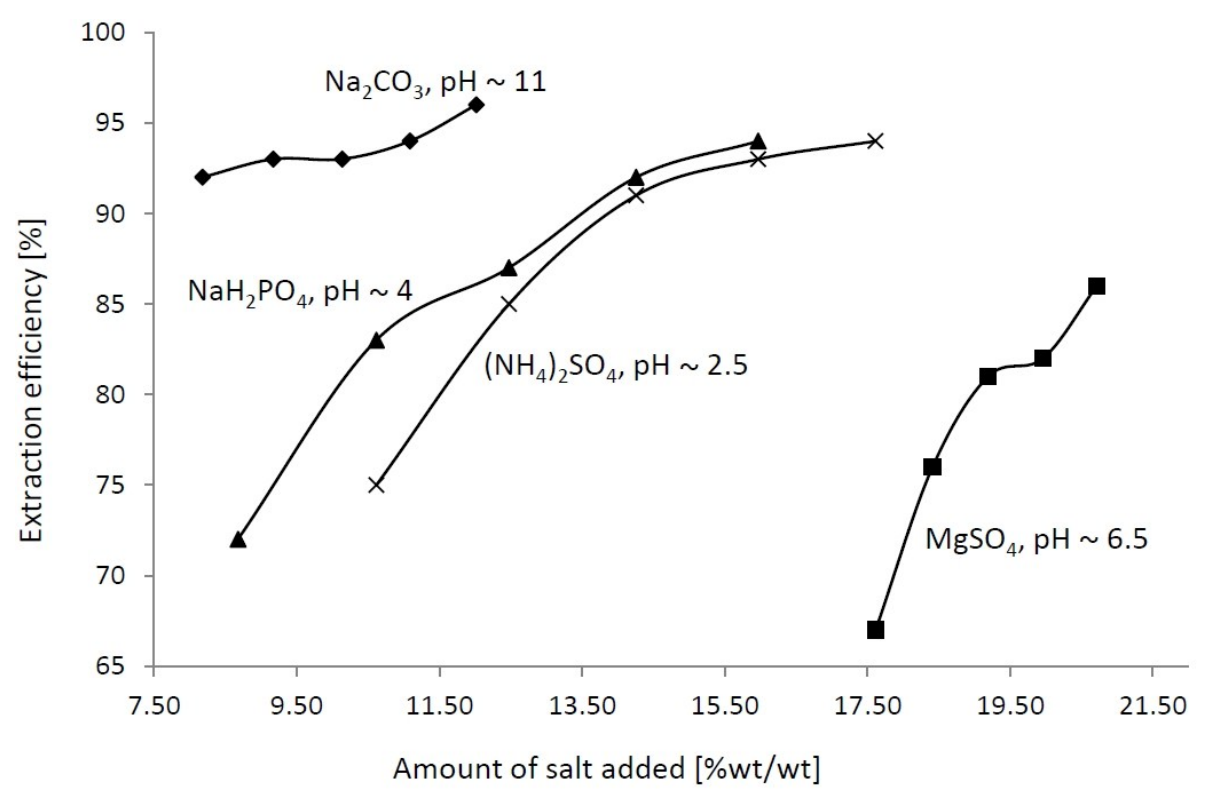

Figure 2. Effect of $\mathrm{pH}$ and salt concentration on the partition of glaucine in IL-ATPS. 
As can be seen, the phase-forming ability of the salts used (represented as an amount of salt added $[\% w t / w t])$ is in the following order: $\mathrm{Na}_{2} \mathrm{CO}_{3}(8.18) \approx \mathrm{NaH}_{2} \mathrm{PO}_{4}(8.68)>\left(\mathrm{NH}_{4}\right)_{2} \mathrm{SO}_{4}(10.62)>>\mathrm{MgSO}_{4}$ (17.61), which is consistent with the Hofmeister series [35]. The partition coefficients, $K$, were found higher than unity regardless of the $\mathrm{pH}$ value of the system, and they increased with the salt concentrations. The data obtained is similar to that previously reported for other alkaloids [32,33], and suggests that the ionic/charge-charge or hydrogen-bonding interactions are not the predominant driving forces for the glaucine partition, since regardless of its charge ( $\mathrm{pKa}=6.4$, Ref. [36]) it preferentially migrates toward the IL-rich phase. Consequently, this phenomenon can be attributed to the enhanced hydrophobic interactions of glaucine with both $\left[\mathrm{C}_{4} \mathrm{C}_{1} \mathrm{im}\right]^{+}$and $[\mathrm{Ace}]^{-}$ions, which includes $\pi-\pi$ or dispersive-type interactions between the aromatic rings or methyl groups of the alkaloid and alkyl side chains of the IL's ions, respectively. This suggestion can be further rationalized by a comparison between the $K$ values of all systems at the first concentration studied. For the $\mathrm{Na}_{2} \mathrm{CO}_{3}$-based system ( $\mathrm{pH} c a .11$ ), where glaucine exists as a base, the observed $K(13.54)$ is nearly three-fold higher compared to the others (5.76, 5.36 and 4.28, $c f$. Table S1), where its protonated form is predominant ( $\mathrm{pH}$ in the range 2.5-6.5). Thus, nearly quantitative extraction of glaucine into the IL-rich phase ( $E E$ of $94 \%$ ) was achieved with the former system compared to the others ( $E E$ in the range $67 \%-75 \%$ ), in a single extractive step. Further increase of the inorganic salt concentration slightly affects the phase ratio $(R)$, but causes simultaneous increase of both $K$ and $E E \%$, the latter showing the better salting-out capacity of the inorganic additives over glaucine and IL.

From the above results, it can be summarized that the glaucine transfer toward the IL-rich phase is facilitated at alkaline $\mathrm{pH}$ and that controlled partition between the two phases by the variation of the $\mathrm{pH}$ value of the system or the inorganic salt type is unachievable. The latter suggests that an additional step for glaucine recovery from the IL-rich phase has to be implemented in order for the target compound to be isolated in pure form. Nevertheless, the results obtained can serve as a platform for the future development of improved procedures for IL recovery from crude plant extracts by its induced separation with a certain inorganic salt. This can be of a great importance from the application standpoint since the removal of water from aqueous solutions accounts for the high production costs of similar industrial processes [25]. To check this, additional preliminary experiments for simultaneous IL and glaucine recovery were conducted. As it can be seen from Figure 2, $\mathrm{Na}_{2} \mathrm{CO}_{3}$ possesses the best phase-forming ability, i.e., ensures phase separation at the lowest amount added. Similarly, it ensures a preferable $\mathrm{pH}$ value (ca.11) for glaucine transfer in a more hydrophobic phase. This way, a tri-phase system consisting of a salt-rich phase, IL-rich phase and butyl acetate, being a more benign organic solvent [13], was formed and the partition of glaucine between the phases and quantity of IL recovered were followed. The study was carried out similarly to the ABS experiments and the volume ratio between the phases was 1:0.8:0.8 in respect to the salt-rich phase. It was found that $c a .90 \%$ of the IL was separated as the IL-rich phase and that $c a .68 \%$ of the target alkaloid was recovered into the organic phase. After separation of the salt-rich and IL-rich phases, the latter one was further extracted twice with fresh portions of butyl acetate (in a ratio 1:1), which resulted in 99\% recovery of the target alkaloid in a purity higher than 95\% (HPLC). In summary, the proposed approach ensures nearly quantitative recovery of glaucine and IL, and about $85 \%$ water removal, after phase separation in a single technological step. 


\section{Conclusions}

The possibility for simultaneous recovery of the biologically active alkaloid $S-(+)$-glaucine and separation of the IL employed for its extraction from Glaucium flavum Cr. (Papaveraceae) by means of IL-ABS was studied. It was found that the target alkaloid is predominantly transferred into the IL-rich phase regardless of influential factors such as $\mathrm{pH}$, salt type and concentration, and that its transfer toward the IL-rich phase is facilitated at alkaline $\mathrm{pH}$. The results obtained were further used as a platform for the development of an improved extractive procedure, ensuring simultaneous glaucine recovery, IL recycling and water removal in a single technological step. Thus, based on the formation of a three-phase system consisting of butyl acetate, an IL-rich phase and a salt-rich phase, nearly quantitative recovery of glaucine ( $>99 \%)$, IL recycling ( $c a .90 \%)$, and water removal ( $c a .85 \%)$ was achieved by salt-induced separation of the crude plant extract.

The results obtained in the present study contribute to the understanding of IL behavior in the extraction processes and suggest a possible application of the developed procedure for the production of natural products at standard conditions. This is of great importance from an industrial point of view, since a considerable amount of time and energy could be economized if such a process is implemented in the technological scheme. In this direction, scale-up experiments are now in progress in our laboratory and the results from the ongoing investigations will be reported in due course.

\section{Acknowledgments}

The financial support of the National Science Fund of Bulgaria at the Ministry of Education and Science (project DFNI T 02/23) is greatly acknowledged. The authors also would like to acknowledge the contribution of the COST Action CM1206-Exchange on Ionic Liquids.

\section{Author Contributions}

All authors contributed equally to this work and have approved the final manuscript.

\section{Conflicts of Interest}

The authors declare no conflict of interest.

\section{References}

1. Anastas, P.T.; Kirchhoff, M.M. Origins, current status, and future challenges of green chemistry. Acc. Chem. Res. 2002, 35, 686-694.

2. Plechkova, N.V.; Seddon, K.R. Applications of ionic liquids in the chemical industry. Chem. Soc. Rev. 2008, 37, 123-150.

3. Smiglak, M.; Pringle, J.M.; Lu, X.; Han, L.; Zhang, S.; Gao, H.; MacFarlane, D.R.; Rogers, R.D. Ionic liquids for energy, materials, and medicine. Chem. Commun. 2014, 50, 9228-9250. 
4. Bogdanov, M.G. Ionic Liquids as Alternative Solvents for Extraction of Natural Products. In Alternative Solvents for NaturalProducts Extraction, Green Chemistry and Sustainable Technology; Chemat, F., Vian Abert, M., Eds.; Springer-Verlag: Berlin, Germany; Heidelberg, Germany, 2014; pp.127-166,

5. Passos, H.; Freire, M.G.; Coutinho, J.A.P. Ionic liquid solutions as extractive solvents for value-added compounds from biomass. Green Chem. 2014, 16, 4786-4815.

6. Lapkin, A.A.; Plucinski, P.K.; Cutler, M. Comparative assessment of technologies for extraction of artemisinin. J. Nat. Prod. 2006, 69, 1653-1664.

7. Carneiro, A.P.; Rodriguez, O.; Macedo, E.A. Separation of carbohydrates and sugar alcohols from ionic liquids using antisolvents. Sep. Purif. Technol. 2014, 132, 496-504.

8. Cláudio, A.F.M.; Neves, M.C.; Shimizu, K.; Canongia Lopes, J.N; Freire, M.G.; Coutinho J.A.P. The magic of aqueous solutions of ionic liquids: Ionic liquids as a powerful class of catanionic hydrotropes. Green Chem. 2015, 17, 3948-3963.

9. Jiao, J.; Gai, Q.Y.; Fu, Y.J.; Zu, Y.G.; Luo, M.; Zhao, C.J.; Li, C.Y. Microwave-assisted ionic liquids treatment followed by hydro-distillation for the efficient isolation of essential oil from Fructus forsythiae seed. Sep. Purif. Technol. 2013, 107, 228-237.

10. Bogdanov, M.G.; Svinyarov, I.; Keremedchieva, R.; Sidjimov, A. Ionic liquid-supported solid-liquid extraction of bioactive alkaloids. I. New HPLC method for quantitative determination of glaucine in Glaucium flavum Cr. (Papaveraceae). Sep. Purif. Technol. 2012, 97, 221-227.

11. Bogdanov, M.G.; Keremedchieva, R.; Svinyarov, I. Ionic liquid-supported solid-liquid extraction of bioactive alkaloids. III. Ionic liquid regeneration and glaucine recovery from ionic liquid-aqueous crude extract of Glaucium flavum Cr. (Papaveraceae). Sep. Purif. Technol. 2015, 155, 13-19.

12. Cláudio, A.F.M.; Ferreira, A.M.; Freire, M.G.; Coutinho, J.A.P. Enhanced extraction of caffeine from Guaraná seeds using aqueous solutions of ionic liquids. Green Chem. 2013, 15, 2002-2010.

13. Ressmann, A.K.; Zirbs, R.; Pressler, M.; Gaertner, P.; Bica, K. Surface-active ionic liquids for micellar extraction of piperine from black pepper. Z. Naturforsch. B J. Chem. Sci. 2013, 68, 1129-1137.

14. Tan, Z.J.; Wang, C.Y.; Yang, Z.Z.; Yi, Y.J.; Wang, H.Y.; Zhou, W.L.; Li, F.F. Ionic Liquid-Based Ultrasonic-Assisted Extraction of Secoisolariciresinol Diglucoside from Flaxseed (Linum usitatissimum L.) with Further Purification by an Aqueous Two-Phase System. Molecules 2015, 20, 17929-17943.

15. Tan, Z.; Li, F.; Xu, X. Isolation and purification of aloe anthraquinones based on an ionic liquid/salt aqueous two-phase system. Sep. Purif. Technol. 2012, 98, 150-157.

16. Cláudio, A.F.M.; Marques, C.F.C.; Boal-Palheiros, I.; Freire, M.G.; Coutinho, J.A.P. Development of back-extraction and recyclability routes for ionic-liquid-based aqueous two-phase systems. Green Chem. 2014, 16, 259-268.

17. Zirbs, R.; Strassl, K.; Gaertner, P.; Schröder, C.; Bica, K. Exploring ionic liquid-biomass interactions: Towards the improved isolation of shikimic acid from star anise pods. RSC Adv. 2013, 3, 26010-26016.

18. Ma, C.H.; Zu, Y.G.; Yang, L.; Li, J. Two solid-phase recycling method for basic ionic liquid [C4mim]Ac by macroporous resin and ion exchange resin from_Schisandra chinensis fruits extract. J. Chromatogr. B 2015, 976-977, 1-5. 
19. Zhu, S.; Ma, C.; Fu, Q.; Hu, L.; Lou, Z.; Wang, H.; Tao, G. Application of ionic liquids in an online ultrasonic assisted extraction and solid-phase trapping of rhodiosin and rhodionin from Rhodiola rosea for UPLC. Chromatographia 2013, 76, 195-200.

20. Petkov, V.; Stancheva, S. In vitro inhibition of Cyclic 3X,5X-AMPc phosphodiesterase by a group of structural analogues of glaucine. Acta Physiol. Pharmacol. Bulgaria 1980, 6, 38-46.

21. Berthe, J.; Remandet, B.; Mazue, G.; Tilson, H.A. Neurobehavioral effects of D-glaucine in rats. Neurobehav. Toxicol. Teratol. 1983, 5, 305-308.

22. Kasè, Y.; Kawaguchi, M.; Takahama, K.; Miyata, T.; Hirotsu, I.; Hitoshi, T.; Okano, Y. Pharmacological studies on dl-glaucine phosphate as an antitussive. Arzneim.-Forsch. 1983, 33, 936-946.

23. Zetler, G. Neuroleptic-like, anticonvulsant and antinociceptive effects of aporphine alkaloids: Bulbocapnine, corytuberine, boldine and glaucine. Arch. Int. Pharmacodyn.Ther. 1988, 296, 255-281.

24. Bogdanov, M.G.; Svinyarov, I. Ionic liquid-supported solid-liquid extraction of bioactive alkaloids. II. Kinetics, modeling and mechanism of glaucine extraction from Glaucium flavum Cr. (Papaveraceae). Sep. Purif. Technol. 2013, 103, 279-288.

25. López-Garzón, C.S.; Straathof, A.J.J. Recovery of carboxylic acids produced by fermentation. Biotechnol. Adv. 2014, 32, 873-904.

26. Bogdanov, M.G.; Petkova, D.; Hristeva, S.; Svinyarov, I.; Kantlehner, W. New guanidinium-based room-temperature ionic liquids. Substituent and anion effect on density and solubility in water. Z. Naturforsch. B J. Chem. Sci. 2010, 65, 37-48.

27. Gutowski, K.E.; Broker, G.A.; Willauer, H.D.; Huddleston, J.G.; Swatloski, R.P.; Holbrey, J.D.; Rogers, R.D. Controlling the aqueous miscibility of ionic liquids: Aqueous biphasic systems of water-miscible ionic liquids and water-structuring salts for recycle, metathesis, and separations. J. Am. Chem. Soc. 2003, 125, 6632-6633.

28. Tan, Z.J.; Li, F.F.; Xu, X.L.; Xing, J.M. Simultaneous extraction and purification of aloe polysaccharides and proteins using ionic liquid based aqueous two-phase system coupled with dialysis membrane. Desalination 2012, 286, 389-393.

29. Tonova, K. Separation of poly- and disaccharides by biphasic systems based on ionic liquids. Sep. Purif. Technol. 2012, 89, 57-65.

30. Deive, F.J.; Rodríguez, A.; Pereiro, A.B.; Araújo, J.M.M.; Longo, M.A.; Coelho, M.A.Z.; Canongia Lopes, J.N.; Esperança, J.M.S.S.; Rebelo L.P.N.; Marrucho, I.M. Ionic liquid-based aqueous biphasic system for lipase extraction. Green Chem. 2011, 13, 390-396.

31. Marques, C.F.; Mourão, T.; Neves, C.M.; Lima, Á.S.; Boal-Palheiros, I.; Coutinho, J.A.; Freire, M.G. Aqueous biphasic systems composed of ionic liquids and sodium carbonate as enhanced routes for the extraction of tetracycline. Biotechnol. Prog. 2013, 29, 645-654.

32. Freire, M.G.; Neves, C.M.S.S.; Marrucho, I.M.; Canongia Lopes, J.N.; Rebelo, L.P.N.; Coutinho, J.A.P. High-performance extraction of alkaloids using aqueous two-phase systems with ionic liquids. Green Chem. 2010, 12, 1715-1718.

33. Freire, M.G.; Teles, A.R.R.; Canongia Lopes, J.N.; Rebelo, L.P.N.; Marrucho, I.M.; Coutinho, J.A.P. Partition Coefficients of Alkaloids in Biphasic Ionic-Liquid-Aqueous Systems and their Dependence on the Hofmeister Series. Sep. Sci. Technol. 2012, 47, 284-291. 
34. Tonova, K.; Svinyarov, I.; Bogdanov, M.G. Hydrophobic 3-alkyl-1-methylimidazolium saccharinates as extractants for L-lactic acid recovery. Sep. Purif. Technol. 2014, 125, 239-246.

35. Shahriari, S.; Neves, C.M.; Freire, M.G.; Coutinho, J.A. Role of the Hofmeister Series in the Formation of Ionic-Liquid-Based Aqueous Biphasic Systems. J. Phys. Chem. B 2012, 116, 7252-7258.

36. Petruczynik, A.; Waksmundzka-Hajnos, M.; Hajnos, M.L. The Effect of Chromatographic Conditions on the Separation of Selected Alkaloids in RP-HPTLC. J. Chromatogr. Sci. 2005, 43, 183-194.

(C) 2015 by the authors; licensee MDPI, Basel, Switzerland. This article is an open access article distributed under the terms and conditions of the Creative Commons Attribution license (http://creativecommons.org/licenses/by/4.0/). 